\title{
Diagnostic value of multislice computerized tomography angiography for aortic dissection: A comparison with DSA
}

\author{
DONG LU ${ }^{1,2}$, CHENG-LI LI ${ }^{1}$, WEI-FU LV ${ }^{2}$, MING NI $^{3}$, KE-XUE DENG ${ }^{2}$, CHUN-ZE ZHOU $^{2}$, \\ JING-KUN XIAO ${ }^{2}$, ZHEN-FENG ZHANG ${ }^{2}$ and XING-MING ZHANG ${ }^{2}$ \\ ${ }^{1}$ Department of Interventional MRI, Shandong Provincial Medical Imaging Research Institute, Shandong University, \\ Jinan, Shandong 250021; ${ }^{2}$ Department of Radiology and ${ }^{3}$ PET/CT Center, Affiliated Anhui Provincial Hospital \\ of Anhui Medical University, Hefei, Anhui 230001, P.R. China
}

Received May 30, 2016; Accepted August 18, 2016

DOI: $10.3892 /$ etm.2016.3985

\begin{abstract}
The aim of the present study was to compare multislice computed tomography angiography (MSCTA) and digital subtraction angiography (DSA) in the diagnosis of aortic dissection. In total, 49 patients with aortic lesions received enhanced computed tomography scanning, and three-dimensional (3D) images were reconstructed by volume rendering (VR), maximum intensity projection (MIP), multiplanar reformation (MPR) and curved planar reconstruction (CPR). The display rate of the entry tear site, intimal flap, true and false lumen from each reconstruction method was calculated. For 30 patients with DeBakey type III aortic dissection, the entry tear site and size of the first intimal flap, aortic maximum diameter at the orifice of left subclavian artery (LSCA), distance between the first entry tear site and the orifice of LSCA, and maximum diameter of aortic true and false lumens were measured prior to implantation of endovascular covered stent-grafts. Data obtained by MSCTA and DSA were then compared. For the entry tear site, MPR, CPR and VR provided a display rate of 95.92, 95.92 and $18.37 \%$, respectively, and the display rate of the intimal flap was $100 \%$ in the three methods. MIP did not directly display the entry tear site and intimal flap. For true and false lumens, MPR, CPR, and VR showed a display rate of $100 \%$, while MIP only provided a display rate of $67.35 \%$. When MSCTA was compared with DSA, there was a significant difference in the
\end{abstract}

Correspondence to: Dr Cheng-Li Li, Department of Interventional MRI, Shandong Provincial Medical Imaging Research Institute, Shandong University, 324 Jingwu Road, Jinan, Shandong 250021, P.R. China

E-mail: w9352472shaozhon@163.com

Dr Wei-Fu Lv, Department of Radiology, Affiliated Anhui Provincial Hospital of Anhui Medical University, 17 Lujiang Road, Hefei, Anhui 230001, P.R. China

E-mail:1wf99@126.com

Key words: aortic dissection, aortography, multislice computed tomography angiography, imaging technique, endovascular-covered stent-grafting display of entry site number and position $(\mathrm{P}<0.05)$, whereas no significant difference was shown in the measurement of aortic maximum diameter at the orifice of LSCA and the maximum diameter of true and false lumens $(\mathrm{P}>0.05)$. In conclusion, among the 3D post-processing reconstruction methods of MSCTA used, MPR and CPR were optimal, followed by VR, and MIP. MSCTA may be the preferable imaging method to diagnose aortic dissection and evaluate treatment of endovascular-covered stent-grafting, preoperatively.

\section{Introduction}

Digital subtraction angiography (DSA) refers to the angiographic images obtained through digital processing and deletion of unwanted tissue images, retaining only the vascular image. This is known as the digital subtraction technique and its characteristics are clear image, and high resolution. It provides a real three-dimensional image through observation of vascular lesions, vascular stenosis position measurement, diagnosis and interventional treatment. DAS is necessary for interventional therapy and suitable for systemic vascular diseases and tumor examination and treatment. The application of DSA diagnosis and interventional treatment for the treatment of cardiovascular diseases has opened up a new field. DSA is regarded as the relative gold standard in the diagnosis of aortic dissection (1).

Multislice computerized tomography angiography (MSCTA) involves the rapid injection of contrast agent via peripheral vein, through the spiral continuous thin layer scanning data obtained in vascular reconstruction and its surrounding structures of three-dimensional images, and diagnosis of a vascular disease, by computer workstation processing. MSCTA is used to diagnose intracranial aneurysm, cerebral venous malformation in the clinic. Additionally, MSCTA can cover regions of interest within a short scan time and compensate the drawbacks of angiography because the reconstructed three-dimensional (3D) images show changes in arterial lumens and lesions in the vascular wall, such as mural thrombosis and calcification, and the relationship between vessels and adjacent tissue or organs (2-4). In particular, the sensitivity of MSCTA for the diagnosis of aortic dissection has been reported to range between 83 and 100\%, and the 
specificity is reported to be $100 \%$. With the prevalent utilization of MSCTA and advancements in image analysis software, patients with suspicious symptoms may be rapidly examined to confirm or eliminate the diagnosis of acute aortic dissection (AAD). Notably, MSCTA may be used to evaluate the original location and range of the intimal tearing and the size of the false lumen and branch-vessel involvement, which are all associated with the management and prognosis of patients (5).

In addition, MSCTA provides assistance in the evaluation of lesion degree, activity, and to some extent, prognosis, providing new options for the diagnosis and treatment of aortic dissection (6). MSCTA and DSA are able to provide important information of accurate visualization of tumor-feeding arteries and draining veins for tumor, with high consistency. DSA was slightly more effective than MSCTA in displaying fine branches. MSCTA is more advantageous in revealing the 3D relationships between the tumor and peripheral vessels. In addition, compared to DSA, a type of invasive diagnostic method, MSCTA is non-invasive, faster and cost-effective. DSA should be used in cases of negative or uncertain findings on MSCTA. However, it has decreased sensitivity in the detection of small outpouchings (7). MSCTA has the potential to replace DSA as the gold standard in the examination of vascular diseases, such as aortic dissection.

In the present study, the value of MSCTA in the diagnosis of aortic dissection through 3D image reconstruction was evaluated and compared with DSA.

\section{Materials and methods}

Clinical data. MSCTA and DSA images of 49 non-consecutive patients with aortic dissection, received in our hospital from December, 2005 to September, 2010 were retrospectively analyzed in the present study. Of the 49 patients, 42 were male and seven were female, with an average age of $49.6 \pm 12.3$ years (range, 35-77 years). Thirty-four patients had hypertension, 11 had diabetes mellitus, six had coronary heart disease and atrial fibrillation, 16 had hepatic and renal inadequacy, two had stroke, two had hydropericardium, 11 had hydrothorax, two had shock and four had renal cysts. Of the 49 patients, 12 were diagnosed via surgery, 30 by DSA, and seven by magnetic resonance imaging, ultrasound or general clinical inspection.

Clinical symptoms. In total, 36 patients presented with 'tearing-like' pains in the chest or abdominal region that radiated towards the back and left upper extremity. Four patients presented with chest distress, shortness of breath or coughing and three patients had ischemic changes in the lower extremities. In the present study, acute aortic dissection $(<48 \mathrm{~h})$ was found in seven patients (14.28\%), subacute aortic dissection ( $>48 \mathrm{~h}$ and $<2$ weeks) in 34 patients $(69.39 \%)$ and chronic aortic dissection (>2 weeks) in eight patients (16.33\%).

\section{Examination methods and post-processing}

MSCTA examination and post-processing. A GE LightSpeed 64 computed tomography (CT) scanner (GE Medical Systems, Milwaukee, WI, USA) was used. The patients were placed in a dorsal position and $100 \mathrm{ml}$ non-ionic contrast agent, Ultravist (iodine $350 \mathrm{mg} / \mathrm{ml}$ ), was injected at 3.5-4.0 ml/sec via the antecubital vein through the use of an automatic high pressure
Table I. Aortic dissection classification of the 49 patients

\begin{tabular}{lcccccc}
\hline Type & \multicolumn{3}{c}{ DeBakey } & & \multicolumn{2}{c}{ Stanford } \\
\cline { 2 - 4 } \cline { 7 - 8 } & I & II & III & & A & B \\
\hline Patients (n) & 16 & 3 & 30 & & 19 & 30 \\
\hline
\end{tabular}

injector. The scan delay in arterial and venous phase scanning was 23-28 and 60-70 sec, respectively. All the patients were scanned from the superior aperture of the thorax to the pubic symphysis. Scanning parameters included $120 \mathrm{kV}$ tube voltage, $280 \mathrm{~mA}$ tube current, $10 \mathrm{~mm}$ slice thickness, $0.8 \mathrm{sec} /$ rot rotation time, table feed $27.5 \mathrm{~mm} / \mathrm{rot}, 1.375: 1 \mathrm{pitch}$, and matrix $512 \times 512$. The acquired data were entered into the Advantage Workstation 4.3 (GE Medical Systems), and the images were reconstructed with a slice thickness of $1.25 \mathrm{~mm}$ and slice interval of $1.25 \mathrm{~mm}$. Post-processing techniques included volume rendering (VR), multiplanar reformation (MPR), curved planar reconstruction (CPR) and maximum intensity projection (MIP).

DSA examination and post-processing. A GE Innova 3100 system (GE Healthcare, Waukesha, WI, USA) was used. A 5F pigtail catheter was placed in the ascending aorta through the femoral artery or radial artery, and two-dimensional DSA was performed. Thirty to forty milliliters of non-ionic contrast agent Ultravist (iodine $350 \mathrm{mg} / \mathrm{ml}$ ) was injected at $15 \mathrm{ml} / \mathrm{sec}$ and $600 \mathrm{PSI}$ pressure using an automatic high pressure injector. Images were obtained in the anterior, posterior, and oblique $30^{\circ}$ projections and where necessary, images in the lateral projection were also taken. The acquired images were developed and post-processed on the Advantage Workstation 4.3 (GE Medical Systems).

Image analysis and evaluation. Images were independently analyzed by two experienced radiologists, using double-blinded methodology. Aortic dissection was further diagnosed in each group of images in terms of initial entry site, re-entry site and size, morphology of true and false lumens, course of intimal flap, branching of the aorta, thrombosis of the true and false lumens, calcification and plaque of the vessel wall, displacement of calcification, and hydropericardium and hydrothorax.

Observational indices. MSCTA and DSA were used to observe the images and scored based on the following criteria: i) Aortic dissection types; ii) morphology and course of true and false lumens as well as presentation of intimal flap; iii) number of intimal entry tears, and the site and size of initial entry; iv) involved condition of aorta branches; and v) complications, such as mural thrombosis, aneurysm, and hydropericardium or hydrothorax.

Evaluation indices. i) Comparison of the presentation of true and false lumens, intimal entry site in the cross-sectional images, MPR, CPR, MIP and VR; and ii) prior to implantation of the endovascular-covered stent-graft, the site and number of entry tears, length of first entry, distance between proximal entry tear and the orifice of left subclavian artery (LSCA), aortic diameter at the orifice of LSCA, the maximum diameter of aortic true and false lumens, regions involving false lumen, 

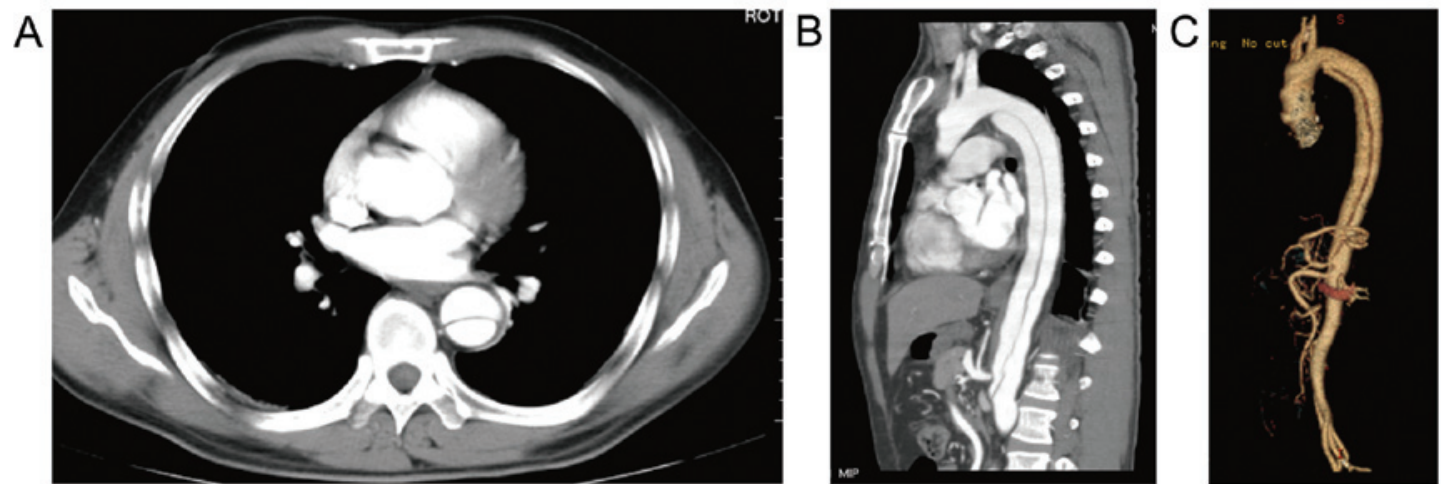

Figure 1. Cross-sectional view: (A) Anterior is true lumen and posterior is false lumen. (B and C) VR and MPR images show that true and false lumens are parallel to the longitudinal axis and there is a linear low-density shadow between the true and false lumens, as shown by the enhanced scan of intimal flap. VR, volume rendering; MPR, multiplanar reformation.
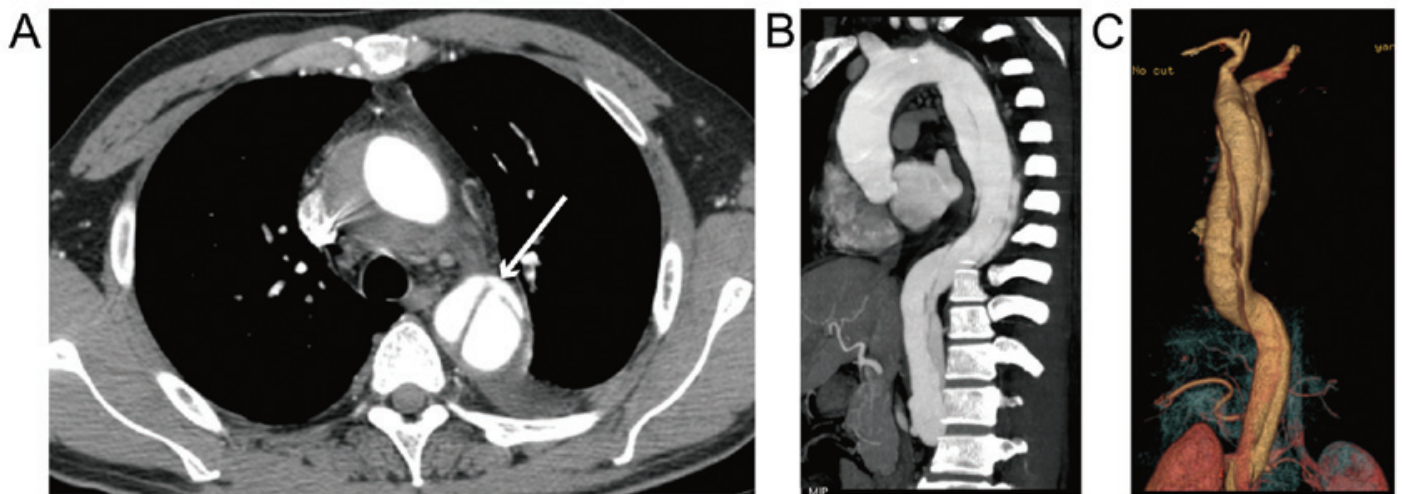

Figure 2. Cross-sectional view: (A) True and false lumens show an irregular morphology, with clear entry tear (white arrow). (B and C) VR and MPR show that true and false lumens travel in a twisting manner. VR, volume rendering; MPR, multiplanar reformation.
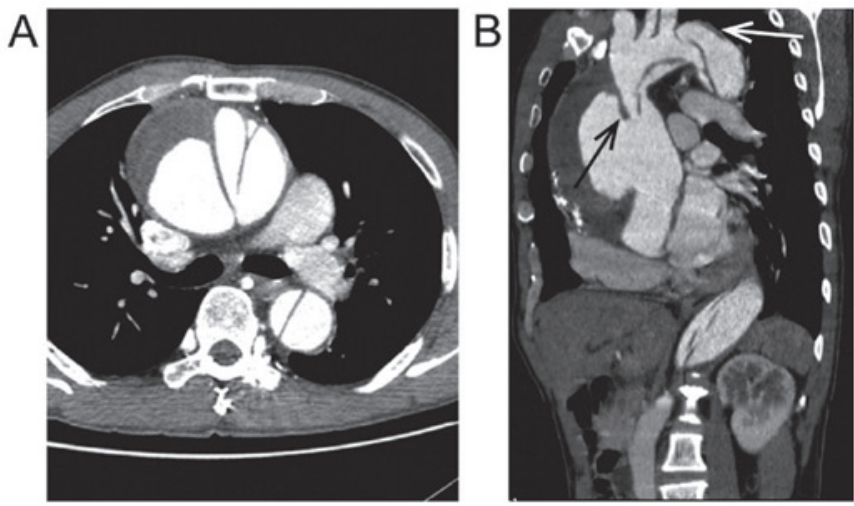

Figure 3. Cross-sectional view: (A) True and false lumens show multi-cavity changes. (B) MPR images demonstrates an initial tear entry in the ascending aorta root (black arrow) and the bow falls (white arrow), respectively. MPR, multiplanar reformation.

the site of second or third entries and the primary branches of abdominal aorta. Following the comparison, the indices were measured and/or evaluated. Data obtained by MSCTA and DSA were compared.

Statistical analysis. Data were entered into Microsoft Excel and statistically analyzed using SPSS 13.0 software (SPSS, Inc., Chicago, IL, USA). Enumeration data were presented as a percentage and analyzed using the $\chi^{2}$ test or Fisher's exact test and pairwise comparison was performed using partitioning $\chi^{2}$. Measurement data were expressed as mean $\pm \mathrm{SD}$. The mean values of two samples were compared using the paired t-test or Wilcoxon rank-sum test. Ranked data were processed using the Wilcoxon rank-sum test. $\mathrm{P}<0.05$ was considered to indicate a statistically significant difference.

\section{Results}

Aortic dissection classification. The precise types of aortic dissection in the 49 patients is shown in Table I.

Morphology and course of true and false lumens and presentation of intimal flap. Of the 49 patients with aortic dissection, the true and false lumens of two patients with DeBakey type III were shown to be parallel to the longitudinal axis (Fig. 1), and the remaining 47 patients exhibited true and false lumens in a twisting manner (Fig. 2). The diameter of the false lumen was larger than that of the true lumen in 37 patients. Furthermore, a true lumen had a diameter of $<1.0 \mathrm{~cm}$ was observed in five patients. Compression of the aortic true lumen by the false lumen was common and observed in 18/37 (48.65\%) patients. There were 12 patients showing similar true and false lumens or true lumens greater than false lumens; in the cross-sectional images, the true lumens appeared ovoid or semicircular and 
Table II. Demonstration of entry tear in 49 patients with aortic dissection using MSCTA.

\begin{tabular}{llccrr}
\hline Entry tear & \multicolumn{1}{c}{ Classification } & $\begin{array}{c}\text { Type I } \\
(\mathrm{n}=16)\end{array}$ & $\begin{array}{c}\text { Type II } \\
(\mathrm{n}=3)\end{array}$ & $\begin{array}{c}\text { Type III } \\
(\mathrm{n}=30)\end{array}$ & $\begin{array}{r}\text { Total } \\
\text { Initial entry tear }\end{array}$ \\
& Ascending aorta & 11 & 3 & 0 & 14 \\
& Aortic arch/isthmus & 3 & 0 & 26 & 29 \\
& Descending thoracic aorta & 0 & 0 & 3 & 3 \\
& Abdominal aorta & 0 & 0 & 1 & 1 \\
\multirow{5}{*}{ Initial entry tears (n) } & Unable to determine precisely & 2 & 0 & 0 & 2 \\
& One & 12 & 3 & 28 & 43 \\
\multirow{3}{*}{ Re-entry tears (n) } & More than one & 2 & 0 & 2 & 4 \\
& Unable to determine precisely & 2 & 0 & 0 & 2 \\
& None & 4 & 0 & 5 & 9 \\
& One & 2 & 2 & 3 & 7 \\
& More than one & 8 & 1 & 22 & 31 \\
\hline
\end{tabular}

MSCTA, multislice computed tomography angiography.

Table III. Demonstration of vessels involved in 49 patients with aortic dissection using MSCTA.

\begin{tabular}{|c|c|c|c|c|c|c|c|c|c|c|c|c|}
\hline Vessel & Type & IA & LCA & LSA & CA & SMA & LRA & RRA & LCIA & RCIA & LIA & RIA \\
\hline \multirow{3}{*}{$\begin{array}{l}\text { False lumen involved } \\
\text { (blood supplied by true } \\
\text { and false lumens) }\end{array}$} & I & 11 & 7 & 2 & 8 & 7 & 8 & 6 & 7 & 10 & 3 & 4 \\
\hline & II & 3 & 1 & 0 & 0 & 0 & 0 & 0 & 0 & 0 & 0 & 0 \\
\hline & III & 0 & 0 & 0 & 11 & 9 & 11 & 7 & 12 & 15 & 2 & 4 \\
\hline \multirow[t]{2}{*}{ Spouting from false lumen } & I & 0 & 1 & 0 & 0 & 0 & 3 & 4 & 0 & 0 & 0 & 0 \\
\hline & III & 0 & 0 & 0 & 2 & 0 & 2 & 5 & 0 & 2 & 1 & 0 \\
\hline Total & & 14 & 9 & 2 & 21 & 16 & 24 & 22 & 19 & 27 & 6 & 8 \\
\hline
\end{tabular}

MSCTA, multislice computed tomography angiography; IA, brachiocephalic artery; LCA, left common carotid artery; LSA, left subclavian artery; CA, celiac artery; SMA, superior mesenteric artery; LRA, left renal artery; RRA, right renal artery; LCIA, left common iliac artery; RCIA, right common iliac artery; LIA, left external iliac artery; RIA, right external iliac artery.
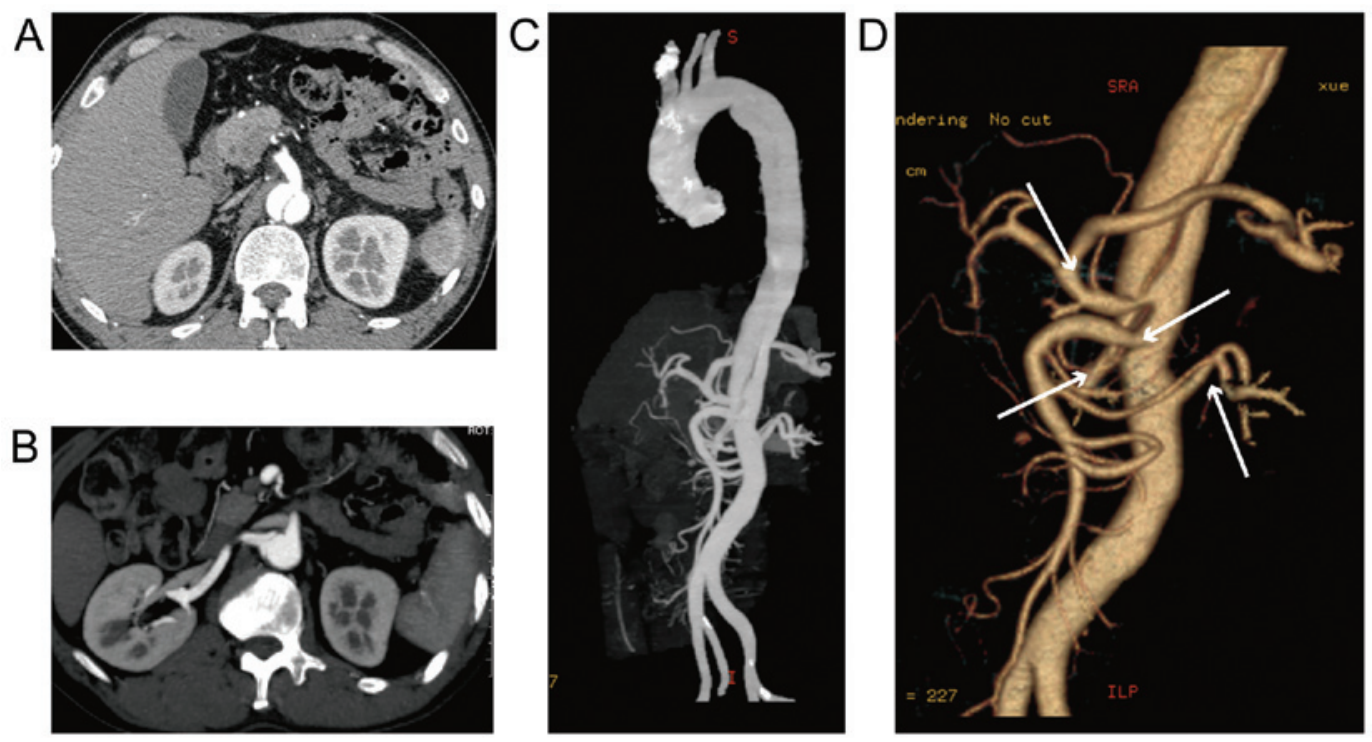

Figure 4. Demonstration of important abdominal vessels involved (cross-sectional view) by VR and MIP images. (A and B) The celiac artery and right renal artery (thin arrow) blood supplied the true and false lumens. (C and D) The superior mesenteric and left renal artery (D, arrow) blood was supplied by the true lumen only. VR, volume rendering; MIP, maximum intensity projection. 

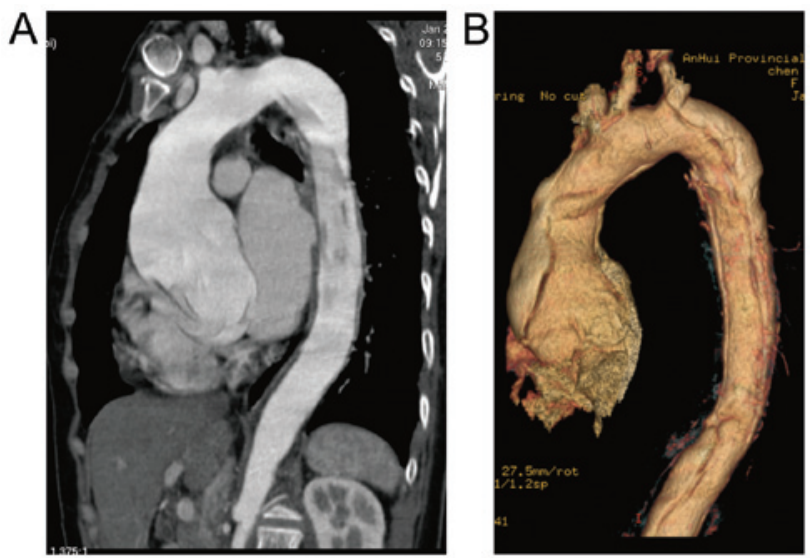

Figure 5. (A) VR and (B) MPR images of a patient with Marfan's syndrome, showing the ascending aortic aneurysm-like ectasia. VR, volume rendering; MPR, multiplanar reformation.

Table IV. Display rate of entry tear, intimal flap and true and false lumens in three-dimensional reconstruction images of aortic dissection $(n=49)$.

\begin{tabular}{lccc}
\hline Group & $\begin{array}{c}\text { Entry tear, } \\
\mathrm{n}(\%)\end{array}$ & $\begin{array}{c}\text { Medial flap, } \\
\mathrm{n}(\%)\end{array}$ & $\begin{array}{c}\text { True and false } \\
\text { lumens, } \mathrm{n}(\%)\end{array}$ \\
\hline MPR & $47(95.92)$ & $49(100)$ & $49(100)$ \\
CPR & $47(95.92)$ & $49(100)$ & $49(100)$ \\
VR & $9(18.37)$ & $49(100)$ & $49(100)$ \\
MIP & 0 & 0 & $33(67.35)$ \\
\hline
\end{tabular}

MRP, multiplanar reformation; CPR, curved planar reconstruction; VR, volume rendering; MIP, maximum intensity projection.

those of the false lumens were crescent-shaped or semicircular. There was a linear low-density shadow between true and false lumens in 49 patients, as shown by the enhanced scan of intimal flap, and in some cases, irregular segmental changes were observed as $\geq 3$ lumens.

Number of entry tears as well as the size and location of initial entry tears. Of the 49 patients with aortic dissection,
47 patients $(95.92 \%)$ showed clear initial entry tear, and in two patients with DeBakey type I, initial entry tears were not found. Of this group, only one initial entry tear was found in 43 patients $(87.76 \%)$, and $\geq 2$ initial entry tears in four patients (8.16\%) (Fig. 3). Thirty-eight patients (77.55\%) had re-entry, of whom 31 had $\geq 2$ re-entries. Re-entry was commonly found in the aortic section. The location and number of initial entry tears as well as the number of re-entries are shown in Table II.

Involvement of vascular branch in aortic dissection. MSCTA showed that the right common iliac artery was involved in aortic dissection $(27 / 49,55.10 \%)$, followed by the left renal artery $(24 / 49,48.98 \%)$, and the LSCA (2/49, 4.08\%). The involved right common iliac arterial blood was usually supplied by the true and false lumens $(25 / 49,51.02 \%)$. The right renal artery was the most common vascular branch in which blood was supplied only by the false lumen $(9 / 49$, 18.37\%) (Table III and Fig. 4).

Other imaging presentations of aortic dissection. Of the 49 patients with aortic dissection, thrombosis of the false lumen was found in 28 patients $(57.14 \%)$; hydrothorax was the most common complication, in 11 patients; hydropericardium in two patients; renal cysts in four patients and aneurysms in nine patients, including one false aneurysm and four aneurysm-like ectasias (Fig. 5). The two types of aneurysm were induced by obvious ectasias of the false lumen.

Demonstration of the true and false lumens, intimal entry, and intimal flap of aortic dissection in $3 D$ reconstruction images. For the entry tear site, MPR, CPR, and VR produced a display rate of $95.92,95.92$ and $18.37 \%$, respectively, whereas MIP did not directly show the entry tear site, and there was a significant difference between them $\left(\chi^{2}=151.149, \mathrm{P}=0.0001\right)$. Partition of the $\chi^{2}$ test for pairwise comparison showed that the display rate of the entry tear site had no statistical difference between MPR and CPR, or between VR and MIP (P>0.05). MPR and CPR provided a higher display rate compared to VR and MIP.

For intimal flap, three of the 3D reconstruction methods discussed above produced a display rate of $100 \%$, although MIP did not directly show the intimal flap. There was a significant difference among the four methods $\left(\chi^{2}=196.000, \mathrm{P}=0.0001\right)$. Partition of the $\chi^{2}$ test for pairwise comparison showed that no
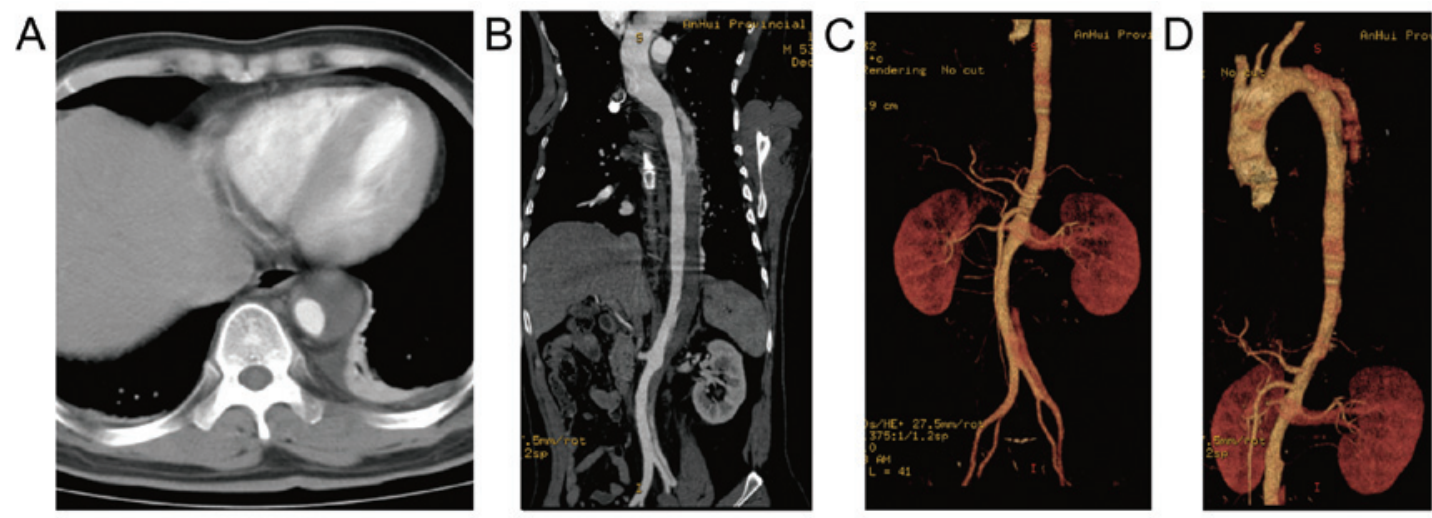

Figure 6. (A) Cross-section images show that the true lumen was small with left hydrothorax, while (B-D) VR and CPR images clearly show the entry tear, intimal flap, true and false lumens, as well as involvement of the left common iliac artery. VR, volume rendering; CPR, curved planar reconstruction. 

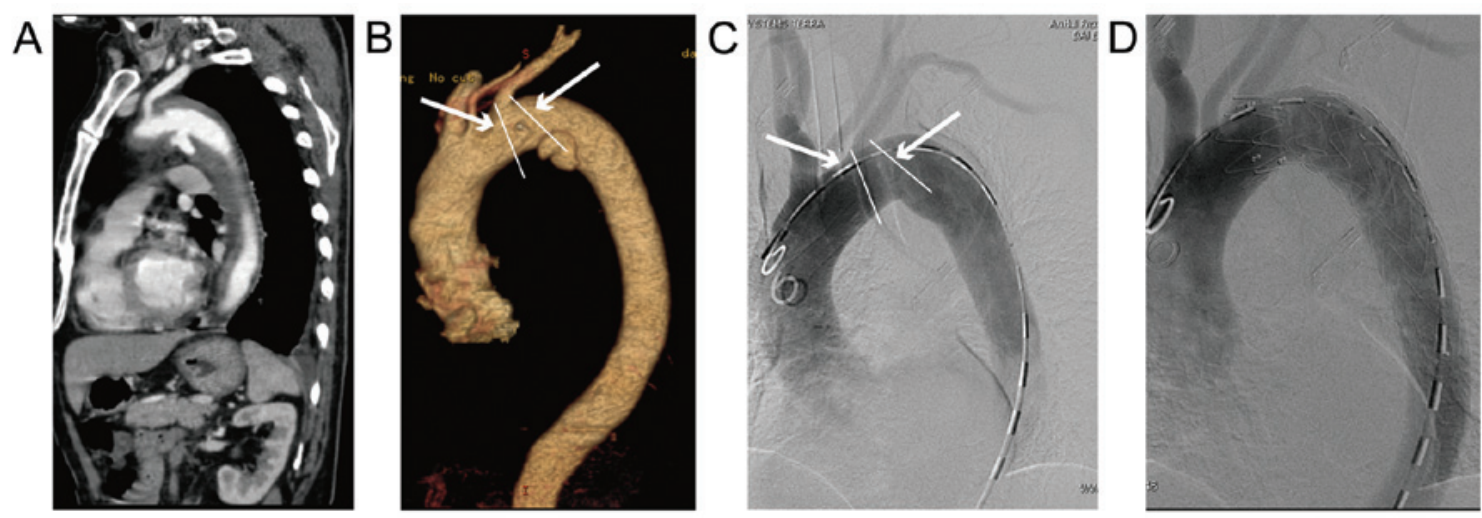

Figure 7. (A and B) Measurement of main parameters using MSCTA. (C and D) DSA prior to implantation of the stent-graft. The thick arrow shows the aortic diameter at the orifice of LSCA and the thin arrow shows the distance between the first tear entry and the orifice of LSCA. MSCTA, multislice computed tomography angiography; DSA, digital subtraction angiography; LSCA, left subclavian artery.

Table V. Comparison of parameters ( $\mathrm{mm})$ in 30 patients with DeBakey type III aortic dissection by MSCTA and DSA.

\begin{tabular}{lcccc}
\hline \multirow{2}{*}{ Item } & \multicolumn{2}{c}{ Mean \pm SD } & & \\
\cline { 2 - 3 } & MSCTA & DSA & $\mathrm{t} / \mathrm{z}$ & P-value \\
\hline $\begin{array}{l}\text { Length of the } \\
\text { first entry tear }\end{array}$ & $1.4 \pm 0.5$ & $1.5 \pm 0.5$ & -0.562 & 0.579 \\
$\begin{array}{l}\text { Distance between } \\
\text { first entry tear }\end{array}$ & $3.5 \pm 2.7$ & $4.2 \pm 2.8$ & -3.114 & 0.002 \\
$\begin{array}{l}\text { and LSCA } \\
\begin{array}{l}\text { Aortic diameter of } \\
\text { LSCA opening site }\end{array}\end{array}$ & $2.6 \pm 0.3$ & $2.7 \pm 0.3$ & -2.006 & 0.054 \\
$\begin{array}{l}\text { Maximum diameter } \\
\text { of true lumen }\end{array}$ & $2.0 \pm 0.5$ & $1.9 \pm 0.5$ & 0.533 & 0.598 \\
$\begin{array}{l}\text { Maximum diameter } \\
\text { of false lumen }\end{array}$ & $1.5 \pm 0.8$ & $1.5 \pm 0.7$ & 1.867 & 0.072 \\
\hline
\end{tabular}

MSCTA, multislice computed tomography angiography; DSA, digital subtraction angiography; LSCA, left subclavian artery.

significant difference was found among MPR, CPR and VR. However, MPR, CPR and VR provided a significantly higher display rate than that of MIP.

For true and false lumens, MPR, CPR, and VR provided a display rate of $100 \%$, while only $67.35 \%$ for MIP. There was significant difference among these four methods $\left(\chi^{2}=52.267\right.$, $\mathrm{P}=0.0001)$. Partition of $\chi^{2}$ test for pairwise comparison showed that there was no significant difference among MPR, CPR and VR, although MPR, CPR and VR provided a significantly higher display rate than that of MIP (Table IV and Fig. 6).

Measurement of parameters in 30 patients with DeBakey type III aortic dissection before endovascular-covered stent-grafting. Statistical analysis demonstrated that MSCTA provided better effects in displaying the distance between the first entry site and the orifice of LSCA than that of DSA. However, there was no significant difference in the measurements of other parameters between MSCTA and DSA $(\mathrm{P}>0.05)$ (Table V and Fig. 7).
MSCTA revealed that one entry tear was found in five of 30 patients with DeBakey type III aortic dissection, two entries in three patients and more than two entries in 22 patients. DSA revealed that one entry tear was found in one patient, two entries in one patient, and two or more entries in 28 patients. There was a statistical difference in the number of entry tears between DSA and MSCTA $(\mathrm{Z}=-2.076, \mathrm{P}=0.038$, Wilcoxon rank-sum test). The involved branch vessel of aortic dissection was shown clearly by MSCTA, the left renal artery was found in 13 patients, including five patients suffering from complicated poor renal perfusion; the right renal artery was found in 12 patients, including six patients having complicated poor renal perfusion; the celiac artery, superior mesenteric artery as well as left and right iliac arteries were found in 13,9, 12 and 17 patients, respectively. Furthermore, seven patients were identified involving the bilateral iliac artery.

\section{Discussion}

The key to CT angiography-mediated diagnosis of aortic diseases is the CT angiography examination technique. MSCTA provides rapid scanning and 3D reconstructed images at any plane that can clearly show the course of fine vascular branches without distortion. Routine scanning from the superior aperture of the thorax to the pubic symphysis is necessary and slice thickness can be adjusted according to the range of lesions in different regions $(8,9)$. The results of the present study have demonstrated that $10 \mathrm{~mm}$ slice thickness and $1.25 \mathrm{~mm}$ slice interval did not produce optimal effects on the quality of aortic MSCTA images, although the X-ray exposure dose was reduced. However, for some target regions, such as renal artery branches and their origins, thin slices were selected, and pitch and slice interval reconstruction was correspondingly adjusted. Some scholars suggested using electrocardiogram gating and modified reconstruction algorithms in CT examination of the aorta to reduce aortic pulsation artifacts and decrease the rate of misdiagnosis $(9,10)$.

Presentations and significance of aortic dissection by MSCTA. The presentations of MSCTA are primarily described as follows (3,8-11): i) Aortic true and false lumens, which are a direct sign of CT diagnosis; ii) a linear low-density 
shadow between true and false lumens resulting from contrast enhancement; iii) discontinuation of the intimal flap at the intimal entry also provides diagnostic value for aortic dissection; iv) aortic intimal calcification ingression, $>5 \mathrm{~mm}$; v) aortic dissection leak or rupture that leads to the formation of hydrops surrounding the aorta or accumulating in the thoracic or abdominal cavity; and vi) thrombosis of the false lumen presents a high-density shadow in the acute phase and low-density shadow in the chronic phase.

Results from the present study have shown that the display rate of the true and false lumens as well as the intimal flap in aortic dissection were $100 \%$. The morphological determination of the true and false lumens in MSCTA of aortic dissection was dependent on the following features: i) The false lumen typically had a larger cross-sectional area and a beak sign; ii) features indicative of a true lumen were outer wall calcification and eccentric intimal flap calcification; iii) at the aortic arch, when one lumen was encircled by another, the inner lumen was invariably the true lumen; iv) cobwebs were present only in the false lumen due to the ribbons of media that were incompletely sheared off by the dissection; and v) the free edge of the intimal flap generally protruded into the false lumen.

Understanding the number of initial entry tears and re-entry as well as entry site in aortic dissection may be useful in the selection of proper treatment regimens. Of the 49 patients with aortic dissection, initial entry was found in 47 patients (95.92\%), including four patients $(8.16 \%)$ having two or more initial entries and re-entry was found in 38 patients $(77.55 \%)$, including 31 patients having two or more re-entries. This information provided effective guidance for the management of re-entries in the implantation of endovascular-covered stent-grafts in these patients.

Aortic dissection involves vascular branches, which may lead to various clinical symptoms of ischemia. One indication of acute dissection is the acute ischemia of organs induced by important vascular branches involved in dissection. Results from the present study showed that the right common iliac artery was usually involved in aortic dissection $(27 / 49,55.10 \%)$, followed by the left renal artery $(24 / 49,48.98 \%)$, and the LSCA $(2 / 49,4.08 \%)$. The blood of the right common iliac artery was supplied by the true and false lumens $(25 / 49,51.02 \%)$ and the blood of the right renal artery was supplied only by the false lumen $(9 / 49,18.37 \%)$.

Of 49 patients with aortic dissection, thrombosis of the false lumen was found in 28 patients $(57.14 \%)$, while thrombosis of the true lumen was not found in any patients. This result was due to blood flowing slowly in the false lumen with a rough inner wall and low intraluminal pressure, during which red blood cells were destroyed and resulted in cell precipitation and eventually blood clotting. Lee et al (12) reported that this symptom can be used to effectively distinguish the true and false lumens with $100 \%$ specificity.

In the present study, there were 11 patients exhibiting hydrothorax, which was the most common complication in this series and was related to the inflammatory reaction. Two patients suffered from hydropericardium resulting from the dissection rupture or leakage; there were nine patients with aneurysms, including one patient with a false aneurysm and four patients with aneurysm-like ectasias. Williams et al (13) conducted an in vitro study showing that changes in the dissection primarily appeared in the false lumen with slight changes in the true lumen, which corresponded with the results of the present study.

Effect of $3 D$ image post-processing techniques on presentation of aortic dissection. General axial-view images can provide a qualitative diagnosis of aortic dissection and are the basis of MSCTA images, while reconstructed 3D images are predominant in showing the pathological changes of aortic dissection and compensate for the shortage of cross-sectional CT images. Therefore, to most efficiently display the true and false lumens as well as the intimal flap of aortic dissection, different display techniques such as axial view images in conjunction with image post-processing or a combination of multiple techniques should be employed (14-17). In the present study, MPR, CPR, MIP and VR methods were used.

MPR and CPR provided a display rate of $95.92 \%$ in the entry tear site and a display rate of $100 \%$ in the intimal flap and the true and false lumens. MIP did not display the entry tear site and the intimal flap, and the display rate of the true and false lumens was only $67.35 \%$. VR was the most effective method of displaying the intimal flap and may provide more intuitive images than MPR and CPR. MPR and CPR allow for the reconstruction of overlaid thin layers, which further reduces the partial volume effect. MPR is better in displaying the aortic root, the positional relationship between mural thrombosis and the vascular lumen, as well as the structure of the outer wall of the vascular lumen, compared with MIP. Thus, MPR is an optional imaging technique, except in the case of axial view images. MIP is the ideal reconstruction technique in CT angiography (14), but provided the worst image effects in displaying aortic dissection in the present study. Therefore, for the diagnosis of aortic dissection, MPR, CPR, MIP and VR are powerful supplementary techniques for axial-view images and each has advantages and disadvantages. In clinical studies, different image post-processing techniques or a combination of various techniques in conjunction with 3D visualization of vascular structure and lesions should be used in different cases.

MSCTA vs. DSA in the diagnosis of aortic dissection. DSA has long been considered the 'gold standard' for the diagnosis of aortic dissection (18). It plays a key role in the treatment of aortic dissection because of its macroscopic, dynamic observation and immediate measurement properties. In this study, aortic dissection as shown by DSA presented aortic widening and wall thickening. In addition, the lesion range, invasion range and the arterial branches whose blood were supplied by the false lumen were clearly visible. DSA can also display aortic regurgitation and provide effective information for determining whether the aortic flap should be managed in ascending aortic dissections. However, DSA often requires aortic angiography of segments, which leads to high doses of contrast medium, thereby increasing the possibility of potential complications. DSA showed a poor capacity to display the calcified plaques on the arterial wall in this study. This result influences the judgment of whether the intraluminal stents are closely attached to the aortic wall, and increases the possibility of internal hemorrhage. 
With the development of intravascular stenting, intraluminal grafting has gradually become the first choice in the treatment of aortic dissection (15). A key to intraluminal graft implantation is to sufficiently evaluate the various parameters of aortic dissection prior to surgery to guide the selection and placement of intraluminal stents and help determine indications and reduce complications.

Comparison of the measurement of intimal entry. Successful surgery depends on evaluating the entry site and size, and number of intimal flaps. The distance between the first entry to the LSCA is very important. Results from the present study have shown that using a high pressure syringe to create high-speed and high-pressure boluses in DSA displayed the entry better than MSCTA. In our experience, tangential projection angiography, especially in the left anterior oblique is useful in the detection of the entry and clearly visualizes the entry site and position, and adjacent vascular branches, in particular the positional relationship between the first entry and the LSCA opening.

Comparison of the measurements of thoracic aorta diameter and entry size. The stents selected should be well matched to the vessels anchored (15-20). Therefore, it is important to select appropriate stent diameters and lengths in the implantation of intraluminal covered stent-grafts $(14,15)$. Because of the first entry of DeBakey type III aortic dissection being situated at the distal end of the LSCA, the measurements of maximal diameter of the thoracic aorta at the LSCA opening and entry size are important for selection of the appropriate diameter and length of stent-grafts. Data measurement is generally performed based on the aortic plane of the LSCA opening site. In the present study, there were no statistical differences in the diagnosis of aortic dissection between MSCTA and DSA. However, DSA only provides two-dimensional information, while MSCTA can use various post-processing techniques to reconstruct images from different directions based on original cross-sectional CT data, which allows for 3D observation and enhances measurement accuracy.

Comparison of the evaluation of true and false lumens. It is highly important to determine certain information prior to surgery, including the invasion range above and below the false lumens, thrombosis, pressure to the true lumens, and whether the important vascular branches are supplied blood by the true lumen (21). In the present study, MSCTA with MPR, MIP, VR, and CPR were used to visualize the aorta, including the course and circulation of the aorta, invasion of important vascular branches, the course and degree of twisting of the true and false lumens, as well as thrombosis in the false lumen, which guided surgical proposals and determined surgical approaches. The results have also shown that MSCTA displayed the perfusion and related complications of invaded organs better than DSA.

In conclusion, among the $3 \mathrm{D}$ post-processing reconstruction methods of MSCTA used in patients with aortic dissection, MPR and CPR were the optimal methods, followed by VR, and MIP. MSCTA may serve as the preferable imaging method to diagnose aortic dissection and evaluate the treatment of endovascular-covered stent-grafting, preoperatively. MSCTA has the potential to replace DSA as the gold standard in the examination of vascular diseases such as aortic dissection.

\section{References}

1. Nikolic IM, Tasic GM, Jovanovic VT, Repac NR, JanicijevicAM, Scepanovic VD and Nestorovic BD: Assessing the quality of angiographic display of brain blood vessels aneurysms compared to intraoperative state. Vojnosanit Pregl 70: 1117-1123, 2013.

2. McMahon MA and Squirrell CA: Multidetector CT of aortic dissection: a pictorial review. Radiographics 30: 445-460, 2010.

3. Kapustin AJ and Litt HI: Diagnostic imaging for aortic dissection. Semin Thorac Cardiovasc Surg 17: 214-223, 2005.

4. Hayter RG, Rhea JT, Small A, Tafazoli FS and Novelline RA: Suspected aortic dissection and other aortic disorders: multi-detector row CT in 373 cases in the emergency setting. Radiology 238: 841-852, 2006.

5. Gu G, Wan F, Xue Y, Cheng W, Zheng H, Zhao Y, Fan F, Han YI, Tong $\mathrm{C}$ and Yao C: Lumican as a novel potential clinical indicator for acute aortic dissection: A comparative study, based on multislice computed tomography angiography. Exp Ther Med 11: 923-928, 2016.

6. Han X, Zhan Y and Chen J: Comparative study of multi-slice CT angiography with digital subtraction angiography in the blood supply of meningiomas. Exp Ther Med 3: 31-36, 2012.

7. Milošević Medenica S1, V Vućković V and Prstojević B: 64-Slice $\mathrm{CT}$ angiography in the detection of intracranial aneurysms: Comparison with DSA and surgical findings. Neuroradiol J 23: 55-61, 2010.

8. Martin ML, Tay KH, Flak B, Fry PD, Doyle DL, Taylor DC, Hsiang YN and Machan LS: Multidetector CT angiography of the aortoiliac system and lower extremities: a prospective comparison with digital subtraction angiography. AJR Am J Roentgenol 180: 1085-1091, 2003.

9. Weber TF, Ganten MK, Böckler D, Geisbüsch P, Kauczor HU and von Tengg-Kobligk H: Heartbeat-related displacement of the thoracic aorta in patients with chronic aortic dissection type B: quantification by dynamic CTA. Eur J Radiol 72: 483-488, 2009.

10. Fleischmann D, Mitchell RS and Miller DC: Acute aortic syndromes: new insights from electrocardiographically gated computed tomography. Semin Thorac Cardiovasc Surg 20: 340-347, 2008.

11. Castañer E, Andreu M, Gallardo X, Mata JM, Cabezuelo MA and Pallardó Y: CT in nontraumatic acute thoracic aortic disease: typical and atypical features and complications. Radiographics 23: S93-S110, 2003.

12. Lee DY, Williams DM and Abrams GD: The dissected aorta: part II. Differentiation of the true from the false lumen with intravascular US. Radiology 203: 32-36, 1997.

13. Williams DM, LePage MA and Lee DY: The dissected aorta: part I. Early anatomic changes in an in vitro model. Radiology 203: 23-31, 1997.

14. Sharma UK, Gulati MS and Mukhopadhyay S: Aortic aneurysm and dissection: evaluation with spiral CT angiography. JNMA J Nepal Med Assoc 44: 8-12, 2005.

15. Ba Diop S, Diop IB, Diakhate CI, Diao M, Alfidja A, Ly Ba A, Ndiaye A, Diop AN, Gueye B, Mbengue A, et al: Spiral CT in aortic dissection. Dakar Med 49: 28-31, 2004 (In French).

16. Xu SD, Huang FJ, Du JH, Li Y, Fan ZM, Yang JF, Yu XY and Zhang ZG: A study of aortic dimension in type B aortic dissection. Interact Cardiovasc Thorac Surg 7: 244-248, 2008.

17. Bellos JK, Petrosyan A, Abdulamit T, Trastour JC and Bergeron P: Retrograde type A aortic dissections after endovascular stent-graft placement for type B dissection. J Cardiovasc Surg (Torino) 51: 85-93, 2010.

18. Lyons J, Gershlick A, Norell M, Rubens M, Magee P and Layton C: Intravenous digital subtraction angiography in the diagnosis and management of acute aortic dissection. Eur Heart J 8: 186-189, 1987.

19. LePage MA, Quint LE, Sonnad SS, Deeb GM and Williams DM: Aortic dissection: CT features that distinguish true lumen from false lumen. AJR Am J Roentgenol 177: 207-211, 2001.

20. Song JW, Li YH, Chen Y, Lu W, Zeng QL, Zhao JB and Mei QL: Endovascular graft exclusion with digital subtraction angiography for treatment of Stanford type B aortic dissection. Nan Fang Yi Ke Da Xue Xue Bao 28: 293-295, 2008 (In Chinese).

21. Lu QS, Jing ZP, Bao JM, Zhao ZQ, Feng X and Zhao J: To resolve the difficulties of entering into the aortic dissections' true lumen in endovascular graft exclusion. Zhonghua Wai Ke Za Zhi 43: 423-425, 2005 (In Chinese). 